\title{
EMERGING FLUX IN ACTIVE REGIONS
}

\author{
MARGARET LIGGETT and HAROLD ZIRIN \\ Solar Astronomy 264-33, California Institute of Technology, Pasadena, CA 91125, U.S.A.
}

(Received 4 July, 1984; in revised form 24 January, 1985)

\begin{abstract}
We have compared the rates at which flux emerges in active and quiet solar regions within the sunspot belts. The emerging flux regions (EFRs) were identified by the appearance of arch filament structures in $\mathrm{H} \alpha$. All EFRs in high-resolution films of active regions made at Big Bear in 1978 were counted. The comparable rate of flux emergence in quiet regions was obtained from SGD data and independently from EFRs detected outside the active region perimeter on the same films. The rate of flux emergence is 10 times higher in active regions than in quiet regions. A sample of all active regions in 31 days of 1983 gave a ratio of 7.5 . We discuss possible mechanisms which might funnel new magnetic flux to regions of strong magnetic field.
\end{abstract}

\section{Introduction}

Although the general appearance of emerging flux in $\mathrm{H} \alpha$ was recognized early by Waldmeier (1955), the important tracer of arch filament systems (AFS) was first recognized by Bruzek (1967, 1969), who coined the term and by Weart nd Zirin (1969), who termed the arch filaments 'bright regions with loops'. It was quickly recognized that these were indeed areas of emerging magnetic flux and the term EFR for emerging flux regions was introduced by Zirin (1971).

Any student of solar activity knows that EFRs often occur in active regions, and that they play an important role in flare production as the newly introduced fields reconnect with existing fields. Since active regions cover only a small fraction of the Sun, the rate of flux emergence there must be relatively high. The phenomenon has received considerable recent attention (Zirin, 1983; Gaizauskas et al., 1983; Martin et al., 1983). Weart (1972) referred to this process as arch filament system (AFS) rejuvenation, and indicated that it happened "several times more often than if the new AFS were just coming up at chance locations". A good example was shown by Zirin (1974, Figure 5); although McMath region 12417 covered a tiny fraction of the Sun, a large dipole, the only EFR on the Sun, came up right in the middle of it, producing substantial change and numerous flares. Similarly, Zirin (1983, Figure 1) shows the Sun with four EFRs of which three are in one active region. Gaizauskas et al. (1983) discuss the development of complexes of activity by continual flux emergence. But none of these discussions attempt statistical analysis of the clumping of EFRs.

In the present work we attempt to make an objective measurement of the rate of flux emergence in active regions relative to the active belts as a whole.

\section{Method and Results}

Although isolated EFRs are easy to count on full disc patrol films, the identification of dipole emergence in existing active regions (ARs) is much more difficult against a 
background of complex fields. These new dipoles can best be detected by examining high-resolution films of active regions. We therefore determined the rate of emergence of EFRs in active regions by studying $\mathrm{H} \alpha$ films from the Big Bear 10 inch telescope, which provides coverage of an area at Sun center of about $4.25 \times 5.5$ arc min. Our major data sample was the full year of 1978 .

To identify a feature positively as an EFR we required that there be an AFS with brightenings at one or both ends and that spots or pores be forming. The association of this type of structure with flux emergence is invariably confirmed by magnetograms and sunspot drawings. In Figure 1 we show a new and growing dipole in an active region recorded in both $\mathrm{H} \alpha$ and videomagnetograms (VMG). The AFSs in $\mathrm{H} \alpha$ match the dipole emergence in the VMG. Figure 2 is an example of one of the smallest EFRs we found arising in an active region. This EFR is considerably larger than our resolution and a smaller (i.e. younger) one would easily have been detected. Thus there is no effect of seeing on the number detected.

While a typical EFR matures in a day or two, some newly developing active regions show continuous flux emergence over a long period. We did not count EFRs in such cases, confining ourselves to mature regions where the original growth had ceased. We also excluded active regions near the limb (greater than $70^{\circ}$ east or west), where detection is difficult. Only if an EFR was in the obvious confines of the active region, i.e. the area of disturbed $\mathrm{H} \alpha$ structure, was it considered to be emerging in an active region. Those outside of this disturbed structure but in the field of view were counted as 'perimeter' EFRs. An estimate of the area of the active regions observed was made so that the rate of EFR appearance per unit area could be determined. The results are given in Table I.

To obtain comparable data on the EFR emergence rate outside active regions was more difficult. Almost all of the $\mathrm{H} \alpha$ films were made in active regions, and what quiet region films we had were often outside the sunspot belts and not strictly comparable. In the few (13) days of quiet Sun observations at BBSO in the sunspot belts, we found 1 EFR. Since the threshold for EFR detection was also lower because of the quiet background we concluded that the sample was not appropriate for use. (However, it does give the same rate of quiet Sun emergence as the other methods.) We therefore used the following methods: First, we utilized the active region numbers reported in Solar Geophysical Data (SGD). The total number of new active regions reported for the year less those identified in SGD as returning regions should indicate the number of regions which originally emerged outside of active regions (390 regions) during that year. We corrected this data by checking several months of observations from our Tel Aviv telescope, with which quiet Sun EFRs are easily seen, to detect EFRs skipped in the SGD count. In 125 days of data, we found 11 additional EFRs or $9 \%$ more than reported by SGD and increased the SGD value accordingly. This corrected value (425) is reported in Table I as "yearly total of new ARs in spot belts".

Second, we counted the EFRs appearing immediately outside of the active regions in the high-resolution movies. This data has the advantage of essentially identical observing technique, and is reported as 'perimeter' in Table I. In 1978 the average field 

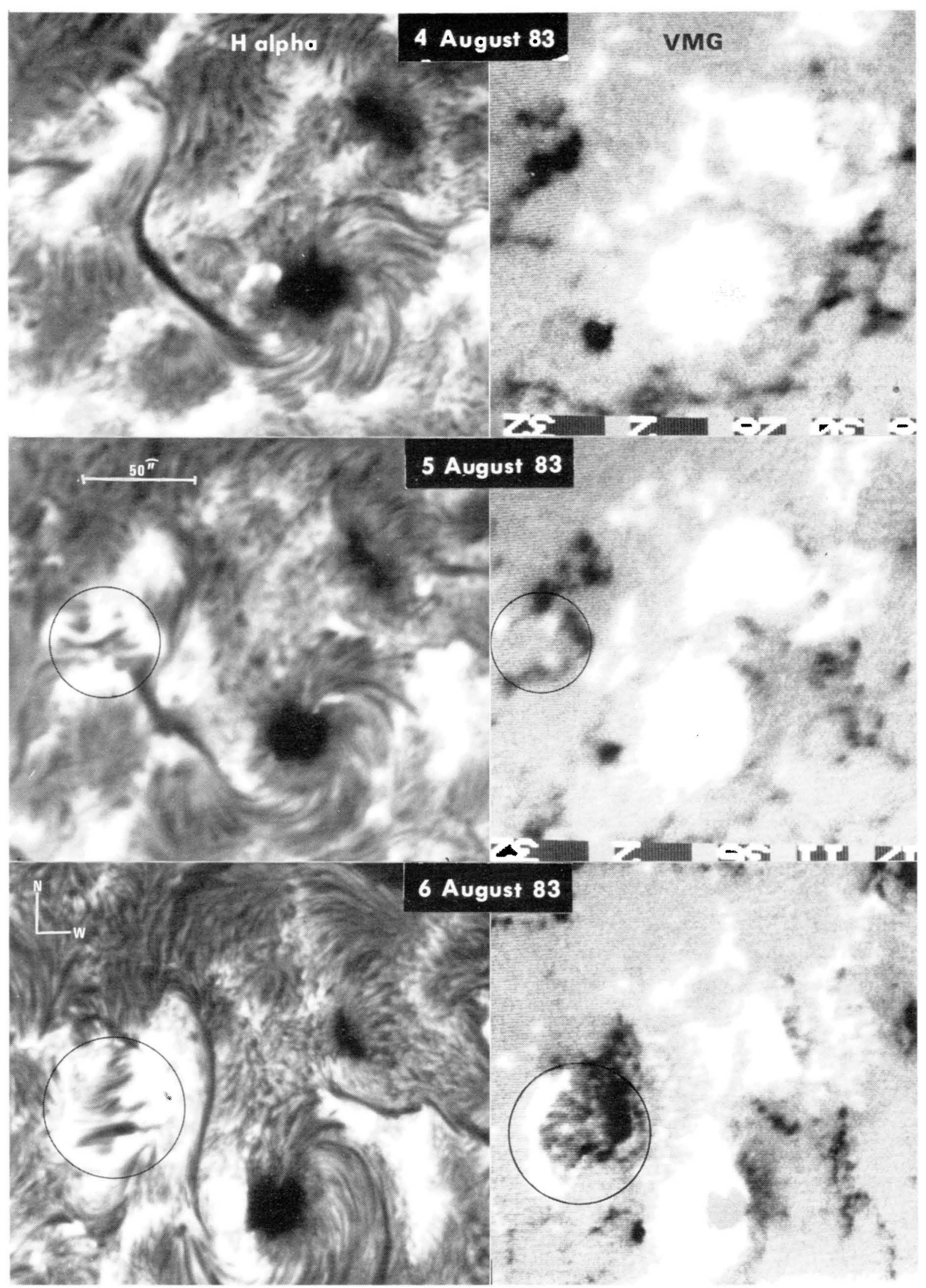

Fig. 1. A new and growing dipole (circled) in an active region recorded in both $\mathrm{H} \alpha$ and videomagnetograms (VMG) illustrates the correlation between arch filament systems (AFSs) and dipole emergence. The AFS in $\mathrm{H} \alpha$ can be seen to relate well to the dipole emergence in the VMG and invariably does so. 

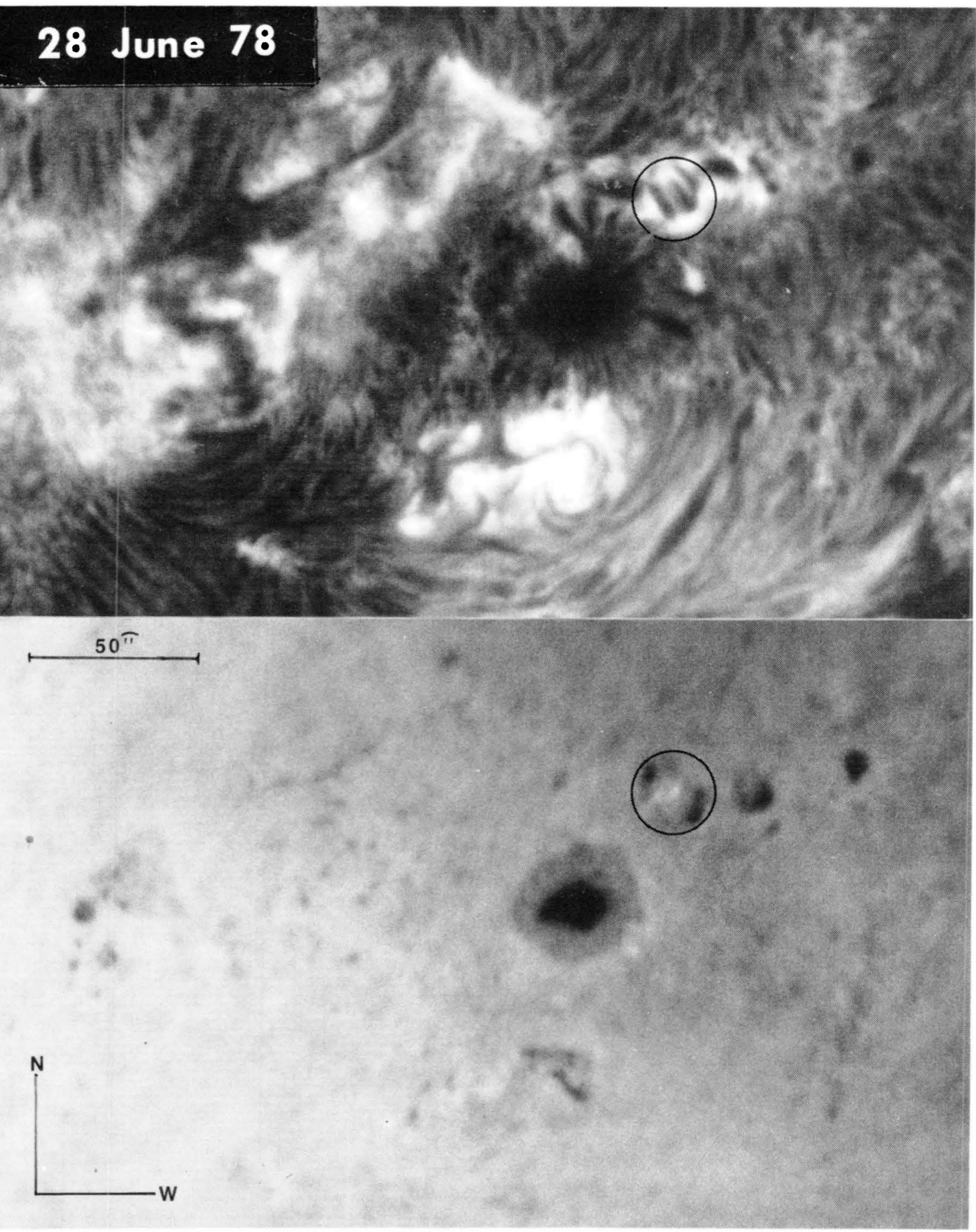

Fig. 2. This illustrates the relative ease in recognizing emerging flux in an active region by AFS identifcation in high-resolution $\mathrm{H} \alpha$ photos. It is one of the smallest emerging flux regions we found arising in an active region. Although the EFR originated overnight, it is already quite well developed. It undoubtedly would have been recognizable at an earlier stage of development and been even smaller in size had we been able to observe it earlier.

of the high-resolution observations was $6.3 \times 10^{10} \mathrm{~km}^{2}$ while the average active region occupied $2.14 \times 10^{10} \mathrm{~km}^{2}$, resulting in a perimeter area of $4.16 \times 10^{10} \mathrm{~km}^{2}$. We see that although the perimeter area was twice that of the active regions, only one tenth as many EFRs were detected. In fact, as one can see in Table I, the 1978 perimeter area seems 
TABLE I

EFR emergence

\begin{tabular}{|c|c|c|}
\hline \multicolumn{3}{|c|}{ Basic data } \\
\hline & 1978 & 1983 \\
\hline Yearly total of new ARs in spot belts & 425 & 257 \\
\hline EFRs in perimeter & 5 & 7 \\
\hline EFRs in ARs & 55 & 31 \\
\hline
\end{tabular}

\begin{tabular}{|c|c|c|}
\hline \multicolumn{3}{|c|}{$\begin{array}{c}\text { Rates } \times 10^{-12} \\
\left(\text { EFR day }{ }^{-1} \mathrm{~km}^{-2} \text { of observed area) }\right.\end{array}$} \\
\hline & 1978 & 1983 \\
\hline Background (spot belts) & 1.34 & 0.62 \\
\hline EFRs in perimeter & 0.63 & 0.77 \\
\hline EFRs in ARs & 13.5 & 4.68 \\
\hline \multicolumn{3}{|c|}{ Ratios } \\
\hline & 1978 & 1983 \\
\hline Background: perimeter & $1: 0.5$ & $1: 1.25$ \\
\hline Background: AR & $1: 10$ & $1: 7.5$ \\
\hline
\end{tabular}

to show a rate of EFR production roughly half that of the background rate determined by the first method.

As can be seen in Table $\mathrm{I}$, in 1978 we observed 55 EFRs emerging in active regions over a span of 190 observing days. This gives a rate of $13.5 \times 10^{-12} \mathrm{EFR}$ day ${ }^{-1} \mathrm{~km}^{-2}$ in 1978 which is 10 times the rate of EFRs produced in the quiet portion (background) of the sunspot belt.

The following data were used in determing these rates: The sunspot belts ranged from about 10 to $30^{\circ}$ latitude. Since we could not properly evaluate any of our film data for regions lying beyond $70^{\circ}$ we calculated an observable area for the sunspot belts of $8.7 \times 10^{11} \mathrm{~km}^{2}$. Each active region and frame area was corrected for foreshortening and then they were averaged together and compared with the total number of EFRs detected in these fields.

The BBSO high resolution observations are often made on the most active region on the Sun, and much of that activity results from the eruption of EFRs. To remove such a bias toward regions of continuous flux eruption we examined a limited sample during two months of 1983 when daily $\mathrm{H} \alpha$ and VMG data on the principal active regions (typically 5) of the disk were obtained. As can be seen in Table $I$, the rate of emergence in active regions in 1983 was only about a third that of the 1978 data. This must be an effect of the sunspot cycle. The background rate of EFR emergence in the belts as a 
whole was also smaller but not quite equivalent, so this independent sample gave a somewhat smaller ratio of active region to background EFR emergence, 7.5:1 compared to $10: 1$ in 1978. Therefore it appears that our 1978 active region sample may indeed have been somewhat biased in favor of high EFR rates.

Background data for this 1983 sample were obtained in a similar fashion to the 1978 study. Since, in 1983, the SGD reports did not indicate whether a region was new or a returning region a $25 \%$ reduction in total number was made based upon a statistical study of active regions by Tang et al. (1984). They determined that of the total number of regions which they studied over a 15 year span, $25 \%$ were returned regions. The 1983 sunspot belts ranged from 0 to $25^{\circ}$ latitude, so the total area (less the area beyond $70^{\circ} \mathrm{E}$ and W) was $1.04 \times 10^{12} \mathrm{~km}^{2}$. The average frame included $6.16 \times 10^{10} \mathrm{~km}^{2}$ of the solar surface and the average active region was $2.60 \times 10^{10} \mathrm{~km}^{2}$. Since the 1983 observing program included an average of 5 regions a day, the daily observed active region area was $1.3 \times 10^{11} \mathrm{~km}^{2}$. The total number of days of observation was 31 .

Despite these checks various other factors affect the comparability of the counts. Sometimes one hemisphere is quite active and the other free of activity. In that case, all the EFRs in the active hemisphere could be considered to erupt in an active region. The absence of the EFRs in the other hemisphere is a global, not active region related phenomenon. In the background counts we count all active regions that emerged, while in the active region data we demanded that more stringent requirements be met before the regions be classified as emerging flux. Further, we only observed about $8 \mathrm{hr}$ per day, but assumed that we would detect any EFRs that came up in the preceding $16 \mathrm{hr}$. The first mentioned condition occurred very rarely in 1978 so had little or no effect on our numbers. The latter factors are more difficult to evaluate. Since their effects would be to increase the rate and we have tried to be as conservative as possible in evaluating this rate, we have chosen not to try to make corrections for them.

The size and lifetime of active regions appear to be strongly dependent on continued flux emergence. The mean life time of sunspot groups is 4 days (Harvey et al., 1975), a number heavily weighted by simple EFRs, which come up and rapidly die if there is no continuous flux emergence. As the initial emergence continues, the group gets larger and larger. But even groups with sizeable, mature spots (with penumbras) do not last more than 10 days without flux emergence. (This is an estimate from experience; we are currently accumulating more accurate statistics.) So if we were mostly observing returning regions we might have been biased in favor of those with a higher rate of flux emergence. To test this idea, we examined the rotation history of the regions observed in 1978 and summarize this in Table II. There are twice as many first rotation as returning ARs listed in SGD whereas we observed almost equal numbers. Thus we, in choosing particularly active regions, had a preference for older ones. These older (i.e. second or later rotation) regions show flux emergence rates nearly twice those of 'younger' regions. That is how they survived that long. Thus it seems likely that the 1983 data study, where all significant active regions were observed, gives a more reliable relative rate ( 7.5 times background) of flux emergence in active regions.

In summary, our data show that the rate of flux emergence is from 7.5 to 10 times 
TABLE II

Active region histories

\begin{tabular}{|c|c|c|c|}
\hline SGD & No. & & $\%$ \\
\hline Total regions in 1978 & 595 & & \\
\hline Number on first rotation ('new') & 390 & & 66 \\
\hline Number on 2nd or greater rotation ('old') & 205 & & 34 \\
\hline Ratio of 'new' to 'old' & $1.9: 1$ & & \\
\hline BBSO & No. & & $\%$ \\
\hline Regions observed in 1978 & 75 & & \\
\hline Number observed to arise on the disc ('new') & 17 & & 22 \\
\hline Number originating on back ('new') & 20 & & 27 \\
\hline Number on 2nd (or $>$ ) rotation ('old') & 38 & & 51 \\
\hline Ratio of 'new' to 'old' & $0.97: 1$ & & \\
\hline EFR emergence & No. & $\%$ & Rate* \\
\hline BBSO ARs with EFRs & 55 & & \\
\hline EFRs arising in 'new' ARs & 12 & 22 & 3.5 \\
\hline EFRs arising in ARs orig. on back & 12 & 22 & 2.8 \\
\hline EFRs arising in AR on 2 nd (or $>$ ) rot. & 31 & 56 & 7.1 \\
\hline
\end{tabular}

greater in active regions than in quiet regions in the active latitudes, and that this flux emergence plays an important role in the longevity of active regions.

\section{Discussion}

One of the remarkable results of this study is how narrowly confined the region of preferred emergence is. As noted above, the areas just outside the active region (perimeters) have rates roughly equivalent to those of the quiet Sun. Why is the emergence rate of EFRs within active regions so great compared to quiet regions? We really don't know. The rate of EFR emergence is proportional to the number of flux loops generated below the surface and the fraction which reach the surface. If the number generated is uniform in the spot belts, then we would have to conclude that flux tubes rise to the surface more easily when there is an interaction with other flux tubes as suggested by Weart (1970). Perhaps the first flux loop makes a path through the convective zone, which otherwise may be hard to penetrate for the young loops. However, this could not account for the wide variation in EFR emergence from region to region. It is possible that some regions have a special structure which encourages emergence of other dipoles. 
One could guess that the new EFRs are additional folds in the original flux loop which eventually reach the surface. However, this would tend to lead to emergence near the active region rather than in it, but we found a low rate of flux emergence in the near neighborhood of ARs.

Perhaps the new EFRs are members of a family of flux loops of which the first is uppermost. This would require a vertical displacement of one loop relative to the other, since the emerging loops are clean dipoles but they interact aggressively with the older ones for surface space. The fact that the formation of sizeable groups requires continuous flux emergence tells us that the subsurface loops can be rather complex.

We should point out that the above process is the major means of formation of delta spots (Tang, 1983). Larger spots of opposite polarity will almost always separate from one another, but spots of opposite polarity belonging to different dipoles will cling together if they get close enough to one another (Zirin, 1983) and form a delta spot which shrinks with time giving up energy in flares. This suggests that many of the secondary EFRs are not part of the original flux loop.

\section{Acknowledgement}

We thank the Big Bear observers, especially for the laborious multi-region imaging in 1983. This work was supported by the NSF under ATM-8211002 and by NASA under NGL 05-002-034.

\section{References}

Bruzek, A.: 1967, Solar Phys. $2,451$.

Bruzek, A.: 1969, Solar Phys. 8, 29.

Gaizauskas, V., Harvey, K., Harvey, J. W., and Zwaan, C.: 1983, Astrophys. J. 265, 1056.

Harvey, K. L., Harvey, J. W., and Martin, S. F.: 1975, Solar Phys. 40, 87.

Martin, S. F., Dezsö, L., Antalová, A., Kučera, A., and Harvey, K. L.: 1983, Adv. Space Res. 2, 39.

Tang, F.: 1983, Solar Phys. 89, 43.

Tang, F., Howard, R., and Adkins, J. M.: 1984, Solar Phys. 91, 75.

Waldmeier, M.: 1955, Ergebnisse und Probleme der Sonnenforschung, Geest and Portig, Leipzig.

Weart, S.: 1972, Astrophys. J. 177, 271.

Weart, S. and Zirin, H.: 1969, Publ. Astron. Soc. Pacific 81, 480.

Zirin, H.: 1971, in R. Howard (ed.), 'Solar Magnetic Fields', IAU Symp. 43, 237.

Zirin, H.: 1974, in R. G. Athay (ed.), 'Chromospheric Fine Structure', IAU Symp. 56, 151.

Zirin, H.: 1983, Astrophys. J. 274, 900. 